\title{
ALTERNATIVE APPROACHES TO IMPROVE PHYSIOLOGICAL PREDICTIONS
}

\author{
Nicholas Oleng', Jaques Reifman \\ Bioinformatics Cell, Telemedicine and Advanced Technology Research Center \\ U.S. Army Medical Research and Materiel Command \\ Frederick, MD 21701 \\ Larry Berglund, Reed Hoyt \\ U.S. Army Research Institute of Environmental Medicine \\ Natick, MA 01760
}

\begin{abstract}
Recent advancements in technology have resulted in new biosensors and information processing capabilities that permit on-line, real-time measurement of physiological variables. This has, in turn, given rise to the possibility of developing soldier-specific, data-driven predictive models for assessing physiological status in the battlefield. This paper explores how the accuracy of a predictive model based on first principles physiology can be enhanced by data-driven "black box" techniques of modeling and predicting human physiological variables. Such hybrid techniques are employed here in the prediction of core temperature. Preliminary results show that the mean square error of prediction can be reduced by up to fifty percent for prediction horizons of up to 30 minutes.
\end{abstract}

\section{INTRODUCTION}

The Warfighter Physiological Status Monitoring (WPSM) program seeks to develop a soldier-wearable, computer-based system for providing commanders and medics with critical physiological status information about their warfighters [Hoyt et. al., 2002; Hoyt and Friedl, 2004]. The WPSM system has two primary aims: the first is to prevent non-battle injuries, such as heat stroke and altitude sickness, and the second is to optimize casualty management through improved casualty detection, diagnostics, and triage. These goals require an array of sensors, a personal area network, and data management software, as well as a variety of decision-support algorithms for monitoring and predicting the soldier's physiologic status. In this paper, we focus on the development of algorithms for nonbattlefield injuries and, in particular, on new techniques that can be used to enhance the accuracy of predictions of important physiological variables.

One of the issues addressed by the WPSM system is the vulnerability of warfighters to heat stroke. There is general consensus that monitoring and/or predicting heart rate and core temperature is an important part of avoiding this preventable non-battle injury. The
SCENARIO model [Kraning and Gonzalez, 1997], a computer simulator developed by the U.S. Army Research Institute of Environmental Medicine (USARIEM), helps perform this task by predicting core temperature, heart rate, and other physiological variables.

Physiological models commonly rely on first-principles knowledge about various mechanisms in the human body and their associated dynamics. The resulting models may be effective in capturing average physiological responses, but not necessarily effective for a particular individual, leading to inaccurate predictions for that individual. The capacity to "tune" a model to a particular individual is particularly important due to the well-documented between-subject variability [Berglund and Stolwijk, 1978; Xu and Werner, 1997]. Individuals with similar anthropomorphic characteristics, subjected to the same work load and environmental conditions, may yield very different physiological responses. Interindividual variation in physiologic response is particularly critical at limiting thresholds of physiologic health, such as extreme values of core temperature, where small variations can make the difference between a suitable recovery and an irreversible pathological condition. A case in point is the approximately 120 heat stroke/sun stroke injuries that occur per year and the associated \$10M/y cost [Sawka et al., 1996; http://amsa.army.mil]. The need to represent betweensubject variability can be addressed by developing models that utilize historic and real-time data that are specific to the individual.

The SCENARIO model, like many other physiological models currently in use, relies primarily on knowledge provided by fundamental first principles of physics and physiology. While the SCENARIO model does incorporate parameters as the individual's weight, height and fat percentage, the fidelity of the model could be improved by accounting for additional relevant dynamics of human physiology. One way of addressing such issues and improving model fidelity, is to incorporate datadriven or "black-box" models into the first-principles model creating "hybrid" models. Such black-box models have been widely used in system identification and 
control in industrial processes and have proven to be quite effective [Ljung, 1999; Goodwin and Sin, 1984]. Physiological dynamics hold equal promise in this regard. Examples of black-box models that could be used in prediction include autoregressive with exogenous input (ARX) models, state-space models, and neural network models.

In the research reported in this paper, we augment the predictions provided by the SCENARIO model with predictions from the black-box models mentioned above. Such hybrid models are expected to account for gaps in understanding of physiological function and betweensubject variability, yielding higher-fidelity predictions than either approach, first-principles or data-driven, can achieve separately.

\section{THE SCENARIO MODEL}

As mentioned in the previous section, SCENARIO is a computer model that seeks to simulate, using first principles, the physiological response of soldiers subjected to environmental and operational stressors. The model simulates the time course of the thermal state of the human body, while considering different environmental conditions, clothing ensembles, work loads, effects of progressive dehydration, and anthropometric characteristics, such as body weight and fat, stature, and varied levels of aerobic fitness. At its core, the model relies on a set of macroscopic energy conservation equations employing a lump-parameter model representation of six adjacent cylindrical compartments that simulate the human body. These are embodied by six differential equations that account for metabolic heat production, passive and active heat exchange between adjacent compartments, and heat loss from the skin and the lungs [Kraning and Gonzalez, 1997].

Although SCENARIO is customizable to an individual, model approximations limit prediction fidelity. For instance, practical considerations necessitate that some of the parameters of the model, such as tissue conductance and metabolic rate, be based on group-average values, instead of values specific to the individual being modeled. Also, since the values of these and other model parameters are based on experimental data, inherent observation error and limited sample size may lead to discrepancies which, compounded, contribute to model inaccuracy. Furthermore, all of the dynamics of human physiology may not have been captured by the SCENARIO model. In order to help address these issues, we propose the incorporation of data-driven, black-box models into the first-principles-based SCENARIO model in the construction of a hybrid modeling approach.

\section{THE HYBRID APPROACH TO MODELING}

Historically, parametric models derived from prior knowledge in the form of empirical correlations, known mathematical equations, or fundamental first principles, such as macroscopic conservation of mass and energy, have been the method of choice in the development of physiologic models. While widely applied, the parametric modeling approach requires comprehensive prior knowledge of the underlying phenomenon being modeled in order to produce accurate predictions. The required accuracy levels often depend of the particular application. Because complete understanding of the underlying phenomenon being modeled is rarely possible, parametric models, such as SCENARIO, often fail to make accurate predictions across the entire spectrum of the model operation. Also, while the model simulates responses occurring during gradual changes of cardiac frequency and core temperature, it needs to respond more accurately to rapid changes in metabolic rates accompanying changes in physical activity and environmental conditions.

On the other extreme, nonparametric models have been proposed to model complex processes when exact analytical equations are unavailable or difficult to develop [Goodwin and Sin, 1984]. These models are derived from process data, where the functional form of the model is conformed to the specifics of the particular process only after the presentation of the data. There are a wide variety of such models ranging from simple ARX models to more complex and nonlinear neural network models. The ARX model [Ljung, 1999] used in our investigation is of the general form

$$
\begin{aligned}
& y(t+d)=\phi^{T}(t) \theta+n(t) \\
& \phi^{T}(t)=\left[y(t), y(t-1), y(t-2), \ldots \ldots . y\left(t-n_{a}\right),\right. \\
& \left.\quad u(t+d-1), \quad u(t+d-2), \ldots \ldots, u\left(t-n_{b}\right)\right]
\end{aligned}
$$

where $y(t)$ represents the output of a model at time $t, d$ corresponds to the prediction horizon, $u(t)$ represents the input at time $t, n(t)$ denotes a white Gaussian noise input, and the regression vector $\phi(t)$ consists of $n_{a}$ past outputs and $n_{b}$ past inputs. Also, $\theta$ denotes a vector of $\left(n_{a}+n_{b}\right)$ parameter coefficients corresponding to these past outputs and inputs.

Figure 1 illustrates the most commonly used artificial neural network, the multi-layer perceptron. In the context of our investigation, this neural network would have only a single output (core temperature) and as many inputs as there are elements of the corresponding regression vector $\phi(t)$ in Equation (1). Thus, the general form may be given as 


$$
\begin{aligned}
y(t+d)=f\left[y(t), y(t-1), y(t-2), \ldots . . y\left(t-n_{a}\right),\right. \\
\left.u(t+d-1), u(t+d-2), \ldots . \quad \ldots ., u\left(t-n_{b}\right)\right]
\end{aligned}
$$

where $f$ is the nonlinear function representing the neural network. Note that in this feed-forward configuration, the output at time $t$ is used as an input to predict the output at the next time step, as illustrated by the dashed line in Figure 1.

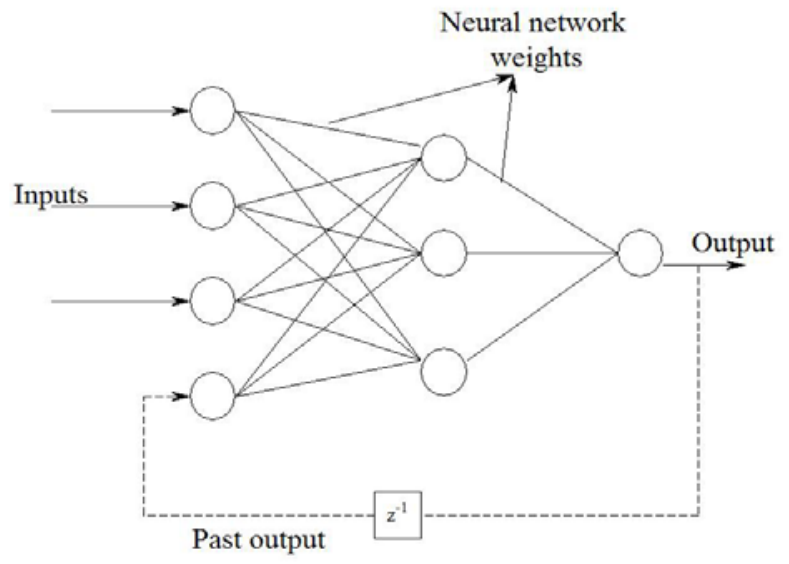

Figure 1: A pictorial representation of a multi-layer feed-forward artificial neural network.

These data-driven models are nevertheless limited to making predictions within the boundaries of the training data. Their ability to extrapolate beyond those limits is unpredictable and their usefulness is extremely dependent on the quantity and quality of the training data, which are often difficult to obtain and validate [Reifman, 2004]. Additionally, as the time horizon of prediction increases for these models, so does the deterioration in the quality of predictions provided by the models. This is because the feedback that could be used to correct for errors in the predictions is not employed until the actual measurement corresponding to that prediction has been made. Thus, for predictions involving large time horizons, predictions based on firstprinciple models may become relatively more reliable.

More recently, hybrid approaches that combine firstprinciples models and the data-driven models mentioned above have been proposed as alternative, more flexible, and perhaps superior modeling paradigms than more traditional parametric and nonparametric approaches [Vilim et al., 2001; Wilson and Zorzetto, 1997]. The promise of hybrid approaches lies in their potential to use the best of both approaches while avoiding the limitations of each approach used separately. Datadriven models complement missing first-principles knowledge with information extracted directly from the process data, while first-principles-based models compartmentalize and reduce the role of data-driven models to specific functionalities, significantly reducing the training data requirements and improving model generalization and extrapolation.

The hybrid approach, when implemented in the context of core temperature prediction involving the SCENARIO model, allows us to employ prior knowledge about the "human physiological process" to the maximum extent possible, and complements the missing knowledge with information extracted from core temperature measurements provided by the array of WPSM biosensors.

The hybrid approach may be implemented under two primary architectures: serial and parallel, as shown in Figures $2 \mathrm{a}$ and $2 \mathrm{~b}$, respectively. The serial approach uses the data-driven model to estimate the parameters of the SCENARIO model, which are then used by SCENARIO to make a prediction of core temperature. The parallel approach, on the other hand, uses the data-driven model to improve the predictions provided by the SCENARIO model. In the following sections we limit our discussions to the latter.

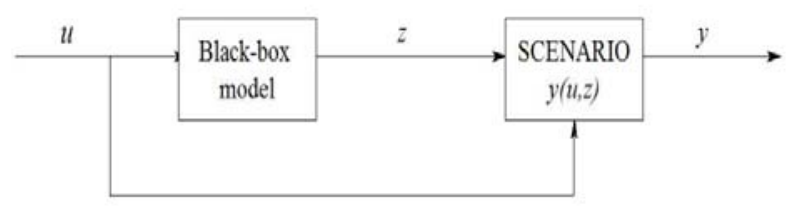

(a)

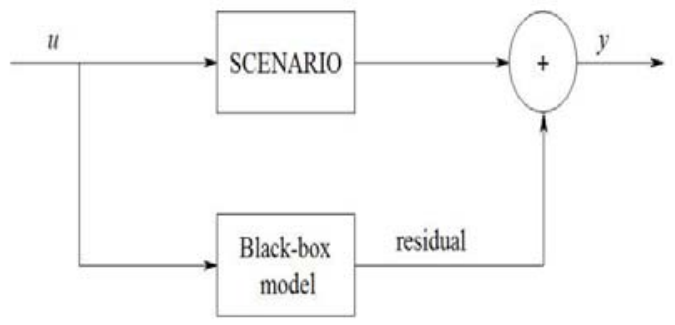

(b)

Figure 2: Two hybrid approaches that combine firstprinciples with data-driven (black-box) models; (a) serial approach and (b) parallel approach.

\section{VALIDATION AND RESULTS}

In this section, we present the results of the implementation of variants of the hybrid model approach to field data using two data-driven models. The particular models implemented here involve: (1) the SCENARIO model in parallel with an ARX model and (2) the SCENARIO model in parallel with a neural network model. 
Seventeen variables corresponding to anthropomorphic and environmental conditions are used as inputs to SCENARIO. These inputs are then processed into 6 variables, corresponding to the six differential equations of the model. It is these 6 variables rather than the 17 inputs that are fed into the black-box model.

Both black-box models, the ARX and the neural network, are employed to estimate the offset between the SCENARIO predictions and the actual core temperature measurements of specific subjects. Accordingly, the resulting prediction of core body temperature is the sum of the predictions provided by the SCENARIO model and that provided by the ARX or the neural network model (Figure 2b).

These models are developed and tested using field data collected from Marines over four days of field training. Each day involved a 3-mile morning march to a shooting range, followed by day-long exercises and rotations within firing stations, and a march back via the same route in the evening. The core temperature for each subject was measured through a telemetry pill ingested at the beginning of every day.

The data from the first day are used to develop (or train) an initial predictive model for each specific individual; that is, to estimate the $\left(n_{a}+n_{b}\right)$ parameter coefficients in Equation (1) and the weights of the neural network in Equation (2). Thereafter, new data from the subsequent three days (which were not previously used to develop the models) are used to refine predictions about future core body temperature in those days. In this paper, we present the results for only one of the subjects, during two of the days.

Figure 3 shows the performance of the ARX hybrid model for one subject over the first two days. The solid (blue) line shows the measured core temperature, sampled once a minute. The thin dashed (green) line shows the predictions provided by the original SCENARIO model while the thick dashed (red) line shows the hybrid model predictions. These predictions are obtained with a time horizon set to 20 minutes. In other words, for each minute, the actual prediction for that minute was computed 20 minutes earlier. The figure also shows the mean square error (MSE) of the prediction for both the hybrid model and the original SCENARIO model. The MSE is defined here as

$$
M S E=\frac{1}{N} \sum_{i} e_{i}^{2}
$$

where $e_{i}$ is the difference between the predicted and the measured temperature at each time instance (i.e., one minute) and $\mathrm{N}$ is the total number of time instances. As can be seen from the figure, the addition of the black-box model to SCENARIO helps improve the accuracy of the 20-minute-ahead prediction during the second day by $50 \%$.

Figure 4 shows the predictions for a 30-minute time horizon. The SCENARIO MSE is unchanged, as this model is based on an open-loop approach that does not rely on feedback from previous predictions or measurements. The hybrid model MSE, however, increases with the time horizon. This is due to the deterioration of the black-box part of the hybrid model as a function of the prediction horizon. In addition, an increase in the time horizon also causes an increased phase shift of the hybrid model prediction. This underscores the importance of the first-principles part of the model as the time horizon increases. An analysis of the precise relation between the prediction time horizon and the resulting phase shift is currently underway.

The linear ARX model described above is not capable of capturing any nonlinear dynamics not incorporated in SCENARIO. Neural networks have been shown to be a useful tool in such cases. Figure 5 shows the results for a 20-minute horizon prediction where a neural network is used as the black-box part of the hybrid model. Surprisingly, while still smaller than the original SCENARIO model, the MSE obtained with the neural network model is larger than that obtained with the ARX model. Additionally, note the increasing number of oscillations in the prediction. While investigations continue, this may point to the possibility that nonlinear dynamics, if present, are insignificant, and that linear models are sufficient in dealing with them. Similar results to those reported here were found when the methods described in this paper were applied to other subjects in the study and during the other two days for which data were available.

\section{CONCLUSIONS AND FUTURE WORK}

The work presented in this paper shows that a hybrid approach (incorporating both first-principle and blackbox models), coupled with closed-loop feedback of prediction errors, improves the prediction accuracy of core temperature for individual subjects. These improvements may reduce the mean prediction error by $20 \%$ to $50 \%$ for prediction horizons varying from 10 to 30 minutes. This has promising implications for many other applications in the arena of physiological modeling and, in particular, future developments of predictive models relevant to the Warfighter Physiological Status Monitoring program.

While the results shown are promising, there still remain many issues to be addressed. Chiefly, the serial hybrid approach to modeling remains to be explored. In this case, a judicious application of the black-box model in improving the estimates of the parameters used in the 

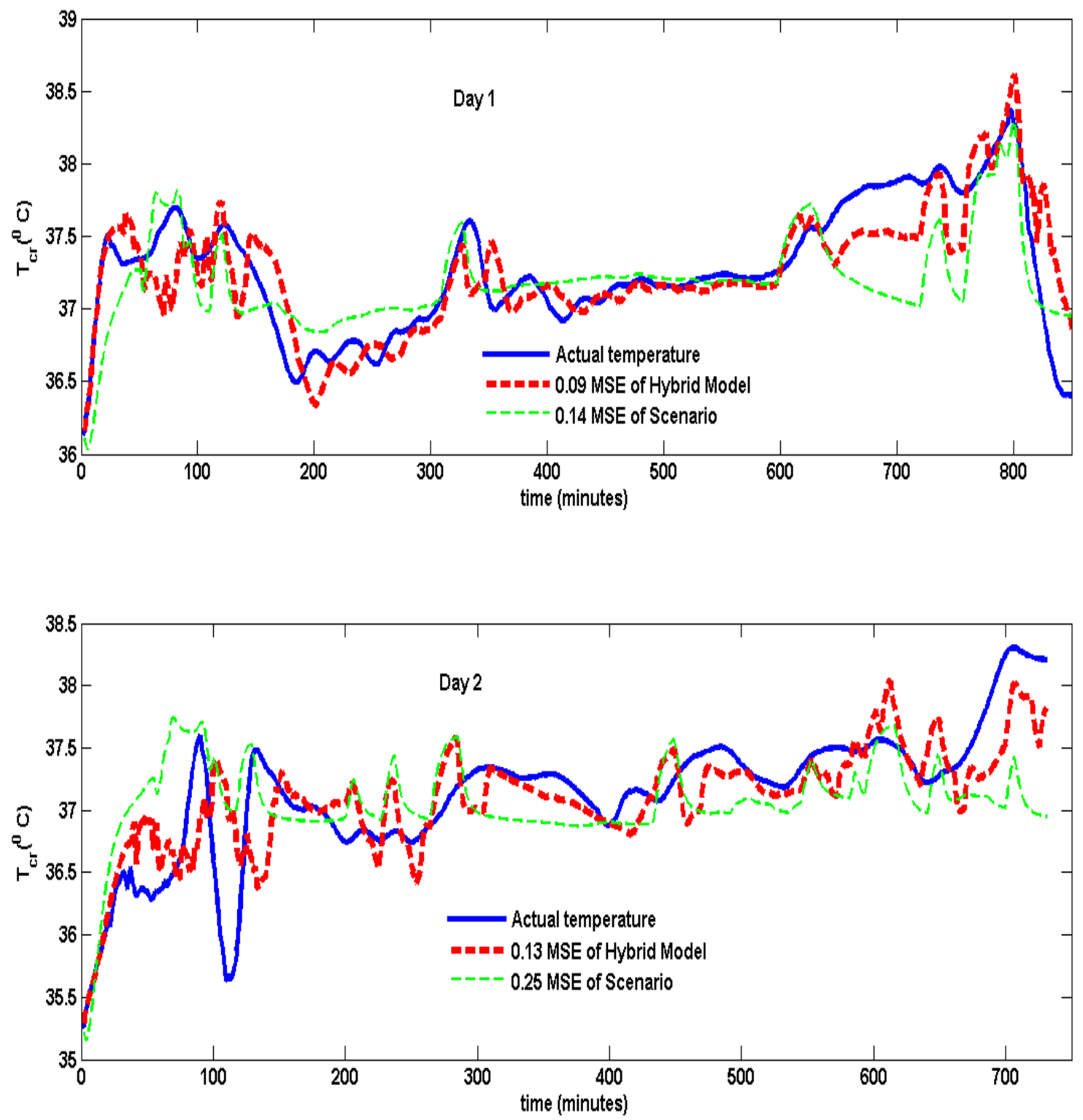

Figure 3: 20-minute ahead prediction of core temperature using an auxiliary ARX model in parallel with SCENARIO.

SCENARIO model would be implemented. Special care must be taken, however, not to mask the insight provided by the SCENARIO model.

Another important issue that needs to be addressed is whether a hybrid model previously developed for one subject may be used as an initial model for a different subject. If so, this would be extremely useful for practical applications, since a subject's data may not always be available for training the model prior to implementation.
The issue of robustness is critical in any modeling application. Therefore, in this context, it is important to have some guarantees of performance. For example, it would be useful to still be able to make a prediction if there is a slight disruption that prevents the measurement of one or more of the variables used in the models.

As is evident from the results presented in this paper, the application of the hybrid approach deteriorates as the prediction horizon increases. In order for decisions to be made on the basis of these predictions, it is critical that 

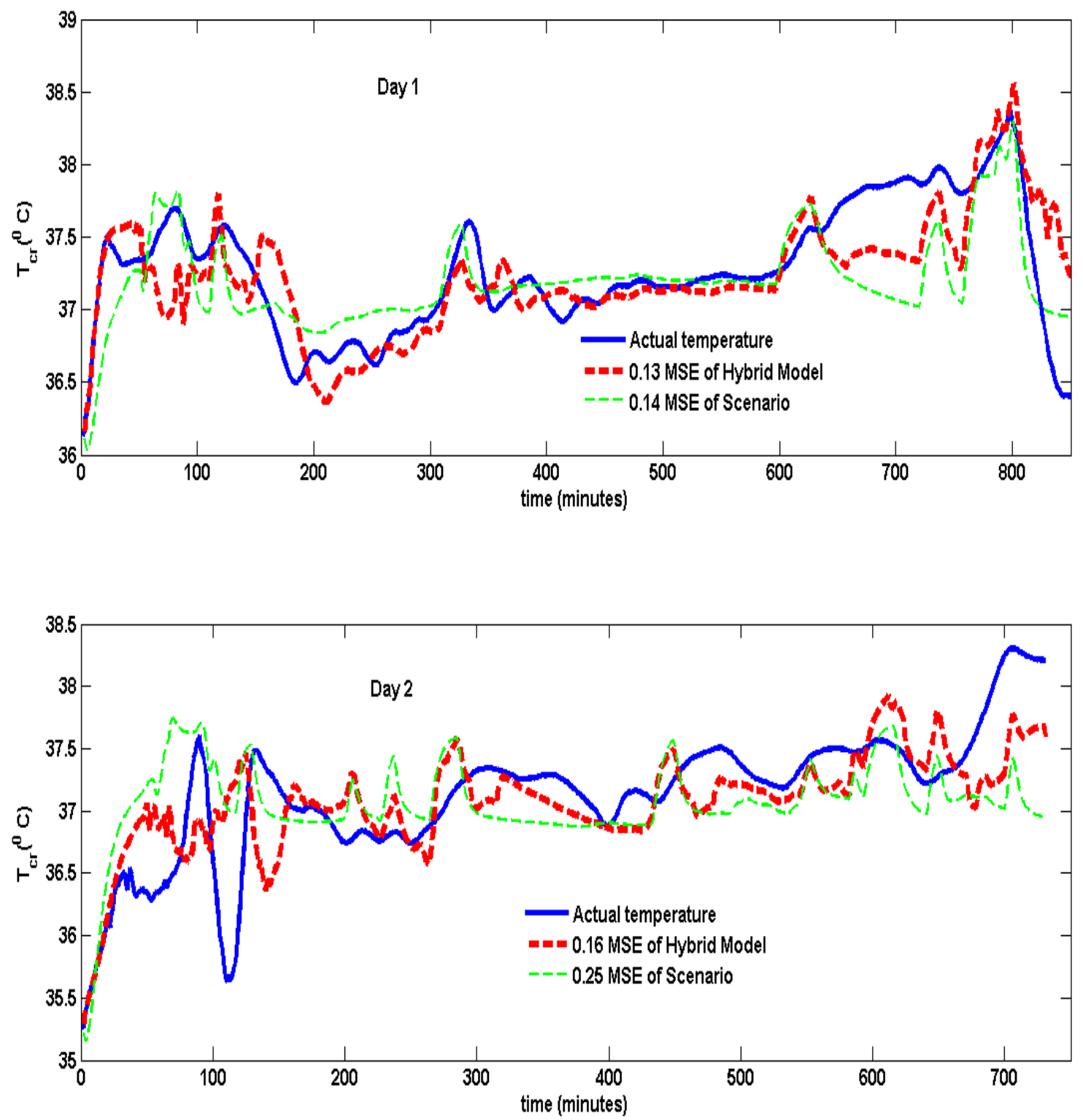

Figure 4: 30-minute ahead prediction of core temperature using an auxiliary ARX model in parallel with SCENARIO.

these predictions be reliable. Hence, it would be in order to quantitatively assess the reliability of the model predictions through estimation of statistical error bounds. One approach that will be pursued in the near future is to apply the statistical bootstrap method [Efrom and Tibshirani, 1993].

While the measurement of body temperature in the study in this paper involved ingestible pill telemetry, future studies will also investigate the effectiveness of using variables such as skin temperature, as alternatives to core temperature, which can be measured in a non-invasive fashion, to monitor and predict heat strain.

\section{ACKNOWLEDGEMENTS}

The authors would like to acknowledge the support of Mark Buller for highly appreciated help with the validation data. The first two authors are supported, in part, by the Combat Casualty Care and the Military Operational Medicine research programs of the U.S. Army Medical Research and Materiel Command. 

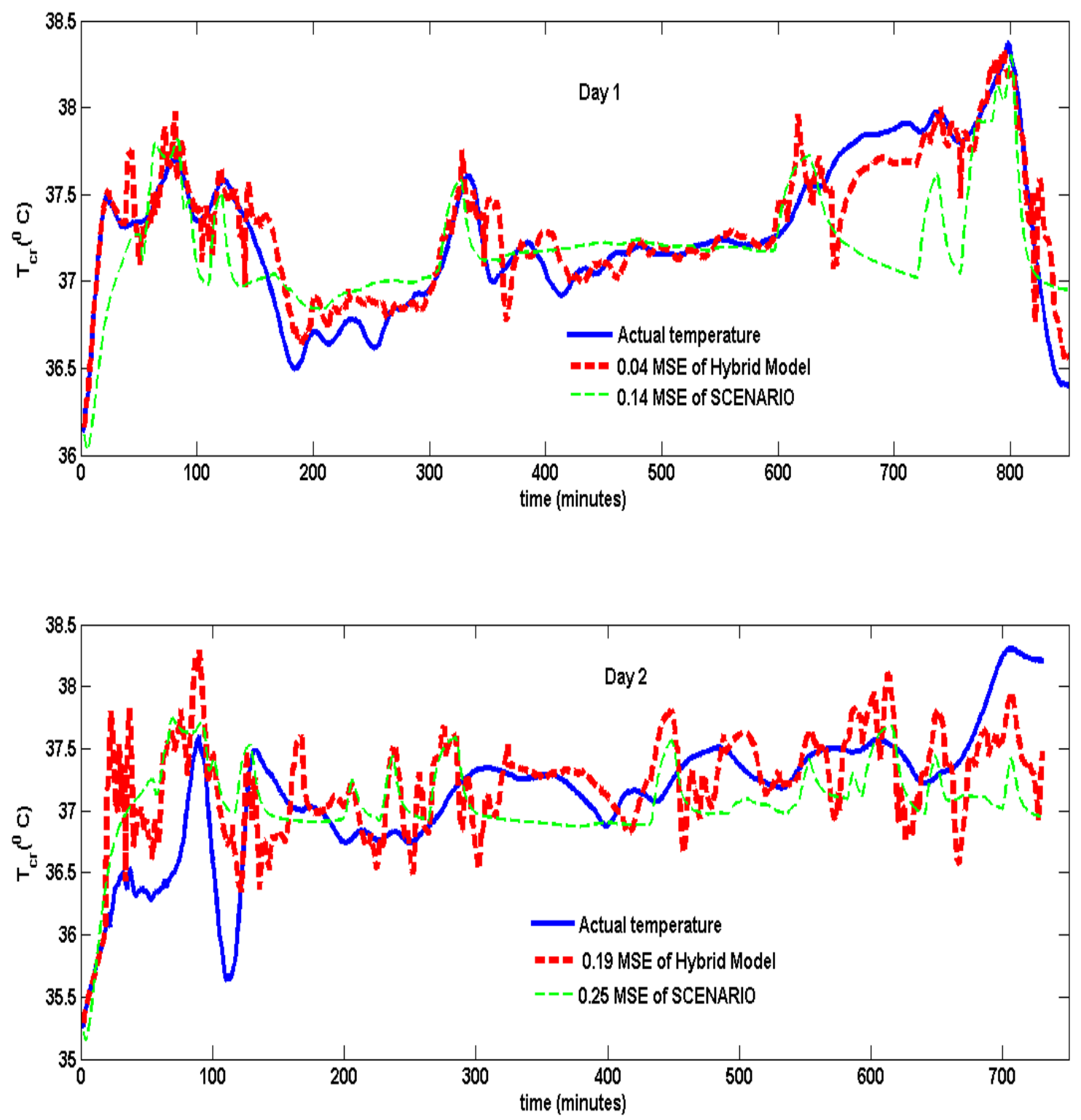

Figure 5: 20-minute ahead prediction of core temperature using an auxiliary neural network model in parallel with SCENARIO.

\section{DISCLAIMER}

In collecting the data presented in this manuscript, the investigators adhered to the policies for protection of human subjects as prescribed in Army Regulation 70-25, and the research was conducted in adherence with the provisions of 45 CFR Part 46 . The subjects gave their informed consent to be studied during their field training exercise after being informed of the purpose, risks, and benefits of the study.

The opinions or assertions contained herein are the private views of the authors and are not to be construed as official or as reflecting the views of the U. S. Army or of the U. S. Department of Defense. 


\section{REFERENCES}

Berglund LG and Stolwijk JAJ.

The use of simulation models of human thermoregulation in assessing acceptability of complex dynamic thermal environments. In: Energy conservation strategies in buildings. Stolwijk JAJ (Ed.). New Haven, CT: Yale Press, 1978, pp.157-191.

Efrom B and Tibshirani RJ.

An Introduction to the Bootstrap, Chapman \& Hall, New York, N.Y, 1993.

Gonzalez RR, McLellan TM, Withey WR, Chang SK and Pandolf KB.

Heat strain models applicable for protective clothing systems: Comparison of core temperature response. J Appl. Physiol. 1997, 83:1017-32.

Goodwin GC and Sin KS.

Adaptive Filtering, Prediction and Control, Prentice Hall, NJ, 1984.

Hoyt, RW and Friedl KE.

Current status of field applications of physiological monitoring for the dismounted soldier. In: Metabolic Monitoring Technologies for Military Field Applications. M. Poos(Ed.). National Academy of Sciences, National Academy Press, 2004, pp. 247-257.

Hoyt RW, Reifman J, Coster TS and Buller MJ.

Combat medical informatics: present and future. In: AMIA 2002 Annual Symposium, pp 335339, San Antonio, Texas, 2002.

Kraning KK and Gonzalez RR.

A mechanistic computer simulation of human work in heat that accounts for physical and physiological effects of clothing, aerobic fitness, and progressive dehydration. J Therm. Biol. 1997, 22:331-42.

Ljung L.

System Identification - Theory for the User, Prentice Hall, NJ 1999.

Reifman J.

Alternative methods for modeling fatigue and performance. Aviation, Space, and Environmental Medicine, 2004, vol.75, No.3, Section II, 173-180.

Sawka MN, Wenger CB and Pandolf KB.

Thermoregulatory responses to acute exerciseheat stress acclimation. In: Handbook of Physiology, Section 4: Environmental Physiology. C.M. Blatteis and M.J. Fregley, (Eds.), New York: Oxford University Press for the American Physiological Society, 1996, Chapter 9, pp. 157-185.

Stolwijk JAJ and Hardy JD.

Control of body temperature. In: Handbook of physiology. Reactions to environmental agents. Lee DHK, (Ed.), Bethesda, MD: American Physiological Society, 1977, pp 45-68.

Wilson JA and Zorzetto FM.

A generalized approach to process state estimation using hybrid artificial neural networks/mechanistic models. Computers in Chemical Engineering 1997, 21:951-63.

Vilim RB, Garcia HE and Chen FW.

An identification scheme combining first principle knowledge, neural networks, and the likelihood function. IEEE Transactions on Control Systems Technology, 2001, 9:186-99.

$\mathrm{Xu} \mathrm{X}$ and Werner $\mathrm{J}$.

A dynamic model of the human/ clothing/ environment - system. Appl. Human Sci., 1997, 16: 61-75. 